\title{
Article \\ Analysis of NOS Gene Polymorphisms in Relation to Cluster Headache and Predisposing Factors in Sweden
}

\author{
Caroline Ran ${ }^{1, *(\mathbb{D}}$, Julia M. Michalska ${ }^{1}\left(\mathbb{D}\right.$, Carmen Fourier $^{1}{ }^{\circledR}$, Christina Sjöstrand ${ }^{2}$, Elisabet Waldenlind ${ }^{2,3}$, \\ Anna Steinberg ${ }^{2,3}$ and Andrea C. Belin ${ }^{1}$ (D) \\ 1 Department of Neuroscience, Karolinska Institutet, 17177 Stockholm, Sweden; \\ juliammichalska@gmail.com (J.M.M.); carmen.fourier@ki.se (C.F.); andrea.carmine.belin@ki.se (A.C.B.) \\ 2 Department of Clinical Neuroscience, Karolinska Institutet, 17177 Stockholm, Sweden; \\ christina.sjostrand@ki.se (C.S.); elisabet.waldenlind@sll.se (E.W.); anna.steinberg@sll.se (A.S.) \\ 3 Department of Neurology, Karolinska University Hospital, 17176 Stockholm, Sweden \\ * Correspondence: caroline.ran@ki.se; Tel.: +46-(0)8-5248-7051
}

check for updates

Citation: Ran, C.; Michalska, J.M.; Fourier, C.; Sjöstrand, C.;

Waldenlind, E.; Steinberg, A.; Belin, A.C. Analysis of NOS Gene Polymorphisms in Relation to Cluster Headache and Predisposing Factors in Sweden. Brain Sci. 2021, 11, 34. https: / / doi.org/10.3390/brainsci 11010034

Received: 8 December 2020 Accepted: 28 December 2020 Published: 31 December 2020

Publisher's Note: MDPI stays neutral with regard to jurisdictional clai$\mathrm{ms}$ in published maps and institutional affiliations.

Copyright: (C) 2020 by the authors. Licensee MDPI, Basel, Switzerland. This article is an open access article distributed under the terms and conditions of the Creative Commons Attribution (CC BY) license (https:// creativecommons.org/licenses/by/ $4.0 /)$.

\begin{abstract}
Cluster headache is characterized by activation of the autonomic-trigeminal reflex. Nitric oxide can trigger headaches in patients, and nitric oxide signaling is known to be affected in cluster headache. Based on the hypothesis of nitric oxide being involved in cluster headache pathophysiology we investigated nitric oxide synthases as potential candidate genes for cluster headache. We analyzed eight variants in the three forms of nitric oxide synthase (NOS) genes, inducible NOS (iNOS), endothelial NOS (eNOS) and neuronal NOS ( $n N O S$ ), and tested for association with cluster headache. Swedish cluster headache patients $(n=542)$ and controls $(n=581)$ were genotyped using TaqMan ${ }^{\circledR}$ assays on an Applied Biosystems 7500 qPCR cycler. This is the largest performed genetic study on NOS involvement in cluster headache so far. We found an association between cluster headache and one iNOS haplotype consisting of the minor alleles of rs2297518 and rs2779249 ( $p=0.022)$. In addition, one of the analyzed $n N O S$ variants, rs2682826, was associated with reported triptan use $(p=0.039)$. Our data suggest that genetic variants in NOS genes do not have a strong influence on cluster headache pathophysiology, but that certain combinations of genetic variants in NOS genes may influence the risk of developing the disorder or triptan use.
\end{abstract}

Keywords: nitric oxide; nitric oxide synthase; neurovascular; smoking; haplotype

\section{Introduction}

Nitric oxide (NO) is a vasodilating molecule and neurotransmitter frequently discussed as a player involved in the pathophysiology of cluster headache $(\mathrm{CH})$. NO is synthesized from L-arginine by means of several NO synthases (NOS). Three genes encode NOS enzymes, neuronal NOS ( NOS or NOS1), inducible NOS (iNOS or NOS2) and endothelial NOS (eNOS or NOS3), which differ by their expression patterns and regulation. iNOS expression is induced by cytokine stimuli in several cell types, namely, macrophages, hepatocytes and smooth muscle cells. eNOS and $n N O S$ rely on the presence of $\mathrm{Ca}^{2+}$ and calmodulin, while $i N O S$ is $\mathrm{Ca}^{2+}$-insensitive [1].

$\mathrm{CH}$ is classified as a trigeminal autonomic cephalalgia, the pathology characterized by excruciatingly painful attacks located around the eye. In active periods, these attacks occur at a frequency of one attack every other day to up to eight attacks per day. Active periods are usually followed by remission periods with no symptoms [2]. During $\mathrm{CH}$ attacks there is an activation of the autonomic-trigeminal reflex, where calcitonin gene-related peptide (CGRP) is released from trigeminal neurons innervating the dura mater, resulting in local inflammation and pain $[3,4]$. The mechanisms involved in triggering a $\mathrm{CH}$ attack remain unknown. NO is involved in many of the biological processes that drive $\mathrm{CH}$ attacks, e.g., vasodilation, inflammation and pain signaling [5,6], and signs of elevated levels of NO activity have been detected in patients [7]. Also, consistent with a role for $\mathrm{NO}$ in $\mathrm{CH}$, NOS 
was shown to be localized to mast cells, nerve fibers and blood vessels in rodent dura mater [8,9]. Changes in NO levels or related molecules could therefore have an impact on $\mathrm{CH}$ pathophysiology.

The activation of the trigeminovascular system that occurs in $\mathrm{CH}$ is also observed in migraine and other primary headache disorders. Similarly, there are several lines of evidence that the involvement of NO could constitute a common pathophysiological feature for several forms of primary headaches. First, nitroglycerin, a compound known as an NO donor (e.g., the metabolization involves liberation of NO), is known to induce headache. When administered to primary headache sufferers (migraine, chronic tensiontype headache or $\mathrm{CH}$ patients), nitroglycerin typically induces a headache with the same characteristics as those usually experienced by the patient, which suggests that NO is a common player in primary headaches [10-12]. Second, several studies investigated NO activity in connection to headache attacks and found signs of elevated NO activity in migraine patients. Increased NO metabolites were detected in the internal jugular blood, venous blood and platelets during a spontaneous migraine attack [13-15]. Furthermore, NO metabolites were suggested to be higher in patients during headache-free periods than in controls, but results are conflicting [16-18]. NO metabolites were additionally found to increase during nitroglycerin-provoked $\mathrm{CH}$ attacks [19]. $\mathrm{CH}$ patients have higher levels of $\mathrm{NO}$ metabolites in the cerebrospinal fluid in comparison to headache-free individuals. In addition, $\mathrm{NO}$ metabolites were also found to be slightly elevated in $\mathrm{CH}$ patients in remission [7]. This is of particular interest in light of the ground-breaking work of Dr. Ekbom, showing that nitroglycerin only triggers $\mathrm{CH}$ attacks in $\mathrm{CH}$ patients who are experiencing an active phase [12]. Together, these studies raise the possibility of a threshold level of NO which triggers a headache attack. Last, a few small candidate gene studies made attempts to investigate single nucleotide polymorphisms (SNPs) and short repeats in NOS genes as risk factors for migraine, so far with inconclusive results. Reports concerning polymorphisms in the $n N O S$ gene showed no association with migraine [20-22]. A study on an $i N O S$ polymorphism similarly lacked association, while a haplotype analysis identified an iNOS haplotype consisting of two markers (rs2779249-rs2297518), which was more common in migraine with aura $[23,24]$. One of the identified SNPs, rs2297518, further correlates with increased risk of migraine in interaction with another variant in eNOS (rs743506) [25]. Results from $e N O S$ studies were also conflicting [26-28]. In concordance with $i N O S$ results, additional associations with migraine with aura were discovered with eNOS haplotype analysis [29]. Genetic studies on NOS and $\mathrm{CH}$ are scarce. There is one previous report on a Swedish case-control material consisting of $91 \mathrm{CH}$ cases and 111 controls, showing an association between a microsatellite marker in iNOS and $\mathrm{CH}$ [30].

As NO signaling and NOS enzymes are appealing candidates for $\mathrm{CH}$, we screened eight markers in these genes and tested for association with $\mathrm{CH}$. Two iNOS markers (rs2297518, rs2779249) and rs743506 in eNOS were included in our study as they were previously linked to migraine. The remaining five markers were selected from published data of associations with vascular disease, as well as SNPs having a possible effect on gene expression and function. We hypothesize that genetic variations in NOS could affect the activity of NOS genes and the production of NO and thereby contribute to the susceptibility of developing $\mathrm{CH}$.

\section{Materials and Methods}

\subsection{Study Material}

The research described herein was performed in accordance with the Declaration of Helsinki. Study material was obtained after approval of the local ethics committee, the Swedish Ethical Review Authority in Stockholm, Sweden (diary number 2014/656-31/4), and receiving informed consent from Swedish $\mathrm{CH}$ patients and neurologically healthy controls living in Sweden. All patients were diagnosed with $\mathrm{CH}$ by a neurologist according to the International Classification of Headache Disorders (ICHD-III beta) criteria [2]. A total of 581 control individuals representing the general Swedish population and $542 \mathrm{CH}$ 
patients were included (Table 1). These were part of a Swedish biobank for $\mathrm{CH}$ which was described previously [31]. Information about clinical aspects, medication and lifestyle was also obtained from the $\mathrm{CH}$ Biobank. The majority of the control individuals were anonymous blood donors; no information, except for sex, could be provided for these individuals.

Table 1. The Swedish case-control material.

\begin{tabular}{ccc}
\hline & Cluster Headache & Controls \\
\hline Number of individuals $(n)$ & 542 & 581 \\
Male $\%$ & 68.5 & 56.6 \\
Chronic subtype $\%$ & 10.1 & NA \\
Average age (years) & 52.3 & NA \\
Age interval (years) & $17-92$ & $18-65$ \\
Age of onset (years) & 31.5 & NA \\
\hline$n=$ number, NA = not available/applicable.
\end{tabular}

\subsection{DNA Isolation}

DNA was purified from whole blood with the Puregene ${ }^{\circledR}$ Blood Core Kit C (QIAGEN Sciences, Hilden, Germany). DNA concentrations were measured with the NanoDrop ${ }^{\circledR}$ ND-1000 Spectrophotometer (NanoDrop Technologies Inc., Wilmington, NC, USA) and the isolated material was used for the screening of selected genetic variants.

\subsection{Genotyping by Quantitative Real-Time Polymerase Chain Reaction}

TaqMan ${ }^{\circledR}$ quantitative Real-Time PCR (qPCR) (Applied Biosystems, Foster City, CA, USA) was used to determine the genotype of different SNPs in a Swedish CH case-control material, with the aim of characterizing susceptibility polymorphisms. Experiments were performed using an ABI 7500 FAST qPCR instrument (Applied Biosystems). PCR plates with 2-5 ng DNA were prepared and dried overnight. TaqMan ${ }^{\circledR}$ SNP Genotyping Assays (Applied Biosystems) containing forward and reverse primers, as well as two $5^{\prime}$ fluorescently labeled (VIC (2-chloro-7phenyl-1,4-dichloro-6-carboxy-fluorescein), FAM (carboxyfluorescein)) allele-specific probes were 1:1 diluted with $1 \times$ TE (Tris EDTA) buffer. The following assays were used: C__15907244_10 (nNOS, rs2682826), C_86363451_10 (nNOS, rs41279104), C_1189257 (iNOS, rs2297518), C_3219460_20 (eNOS, rs1799983), C_115903863_10 (eNOS, rs2070744) and C_30245515_10 (eNOS, rs3918226). For two SNPs, we used custom-made assays previously published by others; 0184316890 (iNOS, rs2779249) and AHFBBRI (eNOS, rs743506) [24,32]. SNP assays were used with TaqMan ${ }^{\circledR}$ Genotyping Master Mix (Applied Biosystems). The qPCR was performed using default fast-cycling conditions: pre-PCR read at $60^{\circ} \mathrm{C}$ for $1 \mathrm{~min}$, holding stage at $95^{\circ} \mathrm{C}$ for $10 \mathrm{~min}, 50$ cycles at $95^{\circ} \mathrm{C}$ for $15 \mathrm{~s}$ and $60^{\circ} \mathrm{C}$ for $1 \mathrm{~min}$; post-PCR read at $60^{\circ} \mathrm{C}$ for $1 \mathrm{~min}$. The above cycling procedure was used for all but one SNP (rs2779249), for which the cycling stage was altered to $92^{\circ} \mathrm{C}$ for $15 \mathrm{~s}$ and $60^{\circ} \mathrm{C}$ for $1.5 \mathrm{~min}$ to increase the time for primer and probe binding, as well as for cleaving of the fluorescent probe by the polymerase. Allelic discrimination was performed during the post-PCR read with the 7500 Software v2.0.6 supplied with the instrument.

\subsection{Statistical Analysis}

Data was analyzed using PLINK v1.07 (http:/ / pngu.mgh.harvard.edu/purcell/plink/) [33]. All SNPs were in Hardy-Weinberg equilibrium for both cases and controls. We used basic allele and genotype testing as well as logistic regression with sex as a covariate to evaluate genotype and allele associations and further verified our data with the Breslow-Day test for homogeneity. Multimarker analysis was performed in PLINK as a separate haplotype analysis for each gene. According to our power analysis (PS-Power and Sample Size Calculations v.3.1.2), we could detect odds ratios (OR) lower than 0.64 or higher than 1.5 with $80 \%$ power for SNPs with a minor allele frequency (MAF) of $20 \%$ in our sample 
size [34]. Stratified analysis for smoking, alcohol consumption and use of verapamil as a prophylactic treatment or triptans to abort attacks was performed in GraphPad Prism v.5.04 (GraphPad Softwares Inc, La Jolla, CA, USA).

\section{Results}

\subsection{Single Marker Analysis}

We genotyped eight NOS gene SNPs in $542 \mathrm{CH}$ patients and 582 control individuals (Table 1) and analyzed these variants for association with increased risk of $\mathrm{CH}$. We performed basic allele and genotypic tests to assess the association for each of the eight genetic variants. Genotypic analysis showed no association between these genetic variants and $\mathrm{CH}$ (data available upon request). Results from the allelic analysis are displayed in Table 2. Our data showed a slight overrepresentation of the minor allele of $i N O S$ variant rs 2779249 in $\mathrm{CH}$ patients. Statistic tests confirmed a weak association between this SNP and $\mathrm{CH}$, which did not remain after correction for multiple testing. Further, allele frequencies in $e N O S$ and $n N O S$ variants as well as rs2297518 in iNOS were equally distributed in $\mathrm{CH}$ patients and controls.

Table 2. Results from the allele analysis for eight variants in endothelial nitric oxide synthase (eNOS), neuronal NOS ( $n N O S$ ) and inducible NOS (iNOS).

\begin{tabular}{|c|c|c|c|c|c|c|c|}
\hline Gene & SNP & Allele & $\begin{array}{l}\text { Controls } \\
\%(n)\end{array}$ & $\begin{array}{c}\text { Cluster } \\
\text { Headache \% (n) }\end{array}$ & $\chi^{2} d f=1$ & $p$-Value & $p_{\mathrm{c}}$-Value \\
\hline \multirow[t]{8}{*}{ eNOS } & rs2070744 & $\mathrm{T}$ & $64.2(723)$ & $65.3(701)$ & 0.27 & 0.60 & 1 \\
\hline & & $\mathrm{C}$ & $35.8(403)$ & 34.7 (373) & & & \\
\hline & rs3918226 & $\mathrm{C}$ & $92.0(1043)$ & $09.0(997)$ & 0 & 1 & 1 \\
\hline & & $\mathrm{T}$ & $8.0(91)$ & $8.0(87)$ & & & \\
\hline & rs1799983 & G & $72.1(826)$ & 71.5 (749) & 1.03 & 0.1 & 0.8 \\
\hline & & $\mathrm{T}$ & $27.9(320)$ & 28.5 (299) & & & \\
\hline & rs743506 & A & $80.5(898)$ & 77.5 (832) & 2.97 & 0.085 & 0.68 \\
\hline & & G & $19.5(218)$ & $22.5(242)$ & & & \\
\hline \multirow[t]{4}{*}{$n N O S$} & rs2682826 & $G$ & $72.6(825)$ & 70.9 (749) & 0.78 & 0.37 & 1 \\
\hline & & A & $27.4(311)$ & $29.1(307)$ & & & \\
\hline & rs41279104 & $\mathrm{C}$ & 86.7 (1007) & 87.8 (938) & 0.68 & 0.41 & 1 \\
\hline & & $\mathrm{T}$ & $13.3(155)$ & $12.2(130)$ & & & \\
\hline \multirow[t]{4}{*}{ iNOS } & rs2297518 & $G$ & $82.7(850)$ & $80.0(818)$ & 2.37 & 0.12 & 0.96 \\
\hline & & A & $17.3(178)$ & $20.0(204)$ & & & \\
\hline & rs2779249 & $\mathrm{C}$ & $71.4(800)$ & $67.6(731)$ & 3.89 & 0.049 & 0.39 \\
\hline & & A & $28.6(320)$ & $32.4(351)$ & & & \\
\hline
\end{tabular}

$\mathrm{SNP}=$ single nucleotide polymorphism, $n=$ number of individuals included in analysis, $\chi^{2} d f=1=$ chi-squared with one degree of freedom, $p_{\mathrm{c}}$-value $=p$-value corrected for eight tests.

As $\mathrm{CH}$ is more common in males than females (68\% males in our material, Table 1$)$, we verified our results using a logistic regression model with sex as a covariate, which showed no significance for these eight SNPs. In order to further verify that the trend for association with rs2779249 was not gender-specific, we ran a Breslow-Day test to analyze for heterogeneity between the male and the female population, but this test showed no variation between the two sexes (data available upon request).

\subsection{Multimarker Analysis}

Previous findings on NOS variants in relation to primary headache disorders showed interesting associations with haplotype analyses in addition to single variants. We therefore ran a haplotype analysis on each of the three NOS genes. We did not find any diseaseassociated haplotypes in $e N O S$ and $n N O S$. Table 3 displays data from the $i N O S$ gene. The haplotype consisting of the minor allele on both positions (AA), was slightly more common in the patient group, with a significant $p$-value of 0.022 . 
Table 3. Results from the rs2297518 I rs2779249 iNOS haplotype analysis.

\begin{tabular}{ccccc}
\hline Haplotype & Frequency in Controls & Frequency in $\mathbf{C H}$ & $\chi^{\mathbf{2}}$ & $p$-Value \\
\hline GC & 0.65 & 0.61 & 2.90 & 0.088 \\
AC & 0.071 & 0.066 & 0.17 & 0.68 \\
GA & 0.18 & 0.19 & 0.25 & 0.62 \\
AA & 0.10 & 0.13 & 5.21 & $\mathbf{0 . 0 2 2}$ \\
\hline
\end{tabular}

GC haplotype $=$ the common allele from both genetic markers, $\mathrm{CH}=$ cluster headache, $\chi^{2}=$ chi-squared with one degree of freedom.

\subsection{Subgroup Analysis Investigating Vasoactive Substances}

As NO is known to act as a potent vasodilator, we further chose to perform a subgroup analysis stratifying for parameters known to affect blood vessel constriction. We used self-reported data obtained from questionnaires filled out by the patients at the time of their inclusion in our biobank [31]. The following parameters were selected for analysis: the use of triptans to abort an attack, use of verapamil as a prophylactic drug, alcohol as a trigger factor for attacks, and coffee and tobacco consumption. Coffee consumption was defined as low (no coffee or less than a cup per day), medium (one to three cups per day) or high (more than four cups per day). Tobacco use was defined as using or having used tobacco in the form of cigarettes or snuff compared to a group of patients having never used tobacco. Use of triptans and verapamil was self-reported at the time of data and material collection. Of the included patients, 51\% reported that alcohol could trigger an attack, $31 \%$ used verapamil as prophylactic medication and $74 \%$ used triptans as acute abortive medication.

When comparing patients using and not using triptans we found an association with the $n N O S$ rs2682826 SNP (Figure 1), $p$-value $=0.039$, (OR 0.72 with a $95 \%$ confidence interval of $0.55-0.98$ ). The minor allele (A) was overrepresented in the group of patients that did not use triptans $(n=140)$ with a minor allele frequency of $33.9 \%$, compared to $27.3 \%$ in patients using triptans $(n=388)$, (for reference, $27.4 \%$ in controls). Coffee, tobacco and verapamil use as well as having alcohol as a trigger factor did not influence the distribution of alleles in patients (data available upon request).

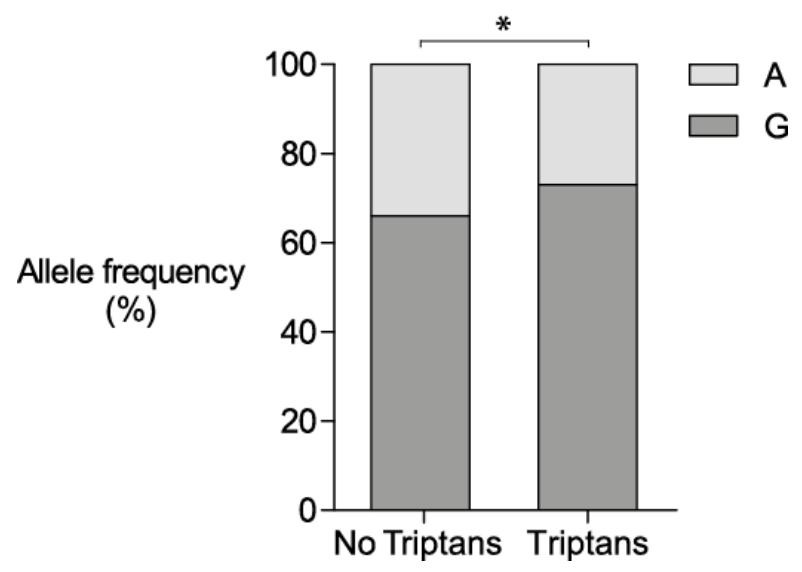

Figure 1. rs2682826 allele frequencies in patients using and not using triptans. Allele frequency of $n$ NOS rs2682826 in triptan users $(n=388)$ vs. nontriptan users $(n=140)$. Analysis showed an association using Fisher's exact test, two-tailed $p$-value, ${ }^{*}=p$-value $<0.05, \mathrm{OR}=0.72,95 \%$ confidence interval $=0.55-0.98$.

\section{Discussion}

The aim of this study was to investigate genetic variants in NOS genes as potential risk factors for $\mathrm{CH}$. We selected eight SNPs for analysis and one showed a trend for association with the disorder (rs2779249 in iNOS). Similar to other NOS candidate gene studies in headache, the strength of the association was insufficient, and significance was 
lost after correction for skewed gender distribution and multiple testing. The sample size in this study was over 500 cases and 500 controls, larger than most genetic studies published previously on NOS genes, but with an OR of 1.2, the discovered association falls outside the range of our $80 \%$ power detection limit $(\mathrm{OR}>1.5$ or $<0.64)$. We performed a haplotype analysis on all three genes, which was in favor of association between $i N O S$ and $\mathrm{CH}$, but not $n N O S$ and $e N O S$. The disease-associated haplotype was composed of two SNPs, both of which are biologically relevant, as they are expected to result in increased transcriptional activity of $i N O S$ (https:/ / gtexportal.org). Although our haplotype analysis supports the association between $i N O S$ and $\mathrm{CH}$, it is likely that the effect of these variants on the risk of developing $\mathrm{CH}$ is relatively small, a conclusion which was also suggested by Sjöstrand et al. in a previous study on NOS variants in Swedish CH patients [30]. Further analyses of these genes in independent cohorts are warranted in order to draw any final conclusions on the genetic contribution of NOS variants in $\mathrm{CH}$. One reason for conflicting and inconclusive results in genetic studies of NOS in headache could be variability due to population background. This was demonstrated previously by Dong et al. in a metaanalysis reporting rs2070744 as a risk factor for migraine only in Caucasians [28]. Secondly, few studies analyzed identical SNPs, contributing to inconsistent results, specifically regarding haplotype analysis.

One potential limitation of our study is the nature of the control group. A majority of the control individuals are anonymous blood donors for whom we have limited information. The blood donors consists of healthy individuals resident in Sweden aged 18-60 years. Therefore, we cannot exclude the possibility that there could be cases of $\mathrm{CH}$ in the control group. However, due to the low incidence of $\mathrm{CH}$, the probability is very small and the occasional occurrence as such should not significantly alter our statistical analysis. In addition, an average of $14 \%$ of our control group might also be considered to suffer from migraine, as this is the prevalence of migraine in the Swedish population. As some of the analyzed SNPs are also associated with migraine, these numbers are more likely to influence our analysis. Referring to allele frequencies in previously published studies, we found that our control population had lower MAFs for all three migraine-associated SNPs (rs2779249, rs2297518 and rs743506), which is why a direct comparison was not possible $[24,25]$. Nevertheless, as the minor alleles of rs2779249 and rs2297518 are associated with increased risk of migraine with aura and the observed relationship between controls of the different studies is inversed [24], we are confident in the validity of our results.

In a subgroup analysis, we identified an association between patients not using triptans and the minor allele of the $n N O S$ SNP rs2682826. Our data do not allow speculations as to why $26 \%$ of our patients do not use triptans. This finding should therefore be interpreted with caution, although we hypothesize that not using triptans is because of lack of efficacy or because of severe side effects. To our knowledge, this is the first indication of a genetic correlation between $n N O S$ and triptan response. rs2682826 is reported as an expression quantitative trait locus (eQTL) for the F-box protein 21 (FBXO21) gene. FBXO21 is a subunit of an ubiquitin protein ligase involved in the innate immune system. Interestingly, a pharmacological compound (NXN-188) with dual activity as an nNOS inhibitor and serotonin 5-HT1B/1D receptor agonist was investigated as a new drug for migraine. The drug was shown to inhibit CGRP release from the dura mater and trigeminal ganglia, but the efficacy of the drug in migraine is still uncertain, and there are no studies on $\mathrm{CH}[35,36]$. In an in vivo study, another nNOS inhibitor, NXN-323, was shown to counteract triptan-induced allodynia in rats. The authors further demonstrated that nNOS expression was increased in dural afferents originating in the trigeminal ganglia after sumatriptan treatment in these rats [37]. Similar events could be at play in the development of triptan-induced medication-overuse headache, suggesting a possible role for nNOS antagonists in future pharmacological treatment strategies for headache. The dynamic interplay of NO and CGRP in the trigeminovascular system was suggested to create an inflammatory loop involving neurons and glial cells, where CGRP released in the trigeminal ganglia increases the production of NO. Released NO further induces CGRP expression and facilitates its 
secretion by trigeminal neurons, thereby creating a feed-forward effect, leading to peripheral sensitization and arterial vessel dilation. It is therefore not unlikely that an optimal treatment effect could be achieved by targeting both pathways. Moreover, genetic predisposition to drug efficacy could well be determined by a combination of common small effect variants, such as rs2682826 in $n N O S$. With the development of personalized medicine, where treatment strategies can be adapted to the individual phenotype and genotype of each patient, knowledge of genetic variants with a potential effect on treatment efficacy are becoming increasingly important. As costs for genetic analysis are rapidly decreasing, evidence-based gene tests for drug screenings could be implemented in clinics in an effort to shorten the time from diagnosis to relief of symptoms for this vulnerable group of patients.

\section{Conclusions}

In conclusion, we performed the largest genetic analysis on NOS involvement in $\mathrm{CH}$ so far. Our results indicate that combinations of genetic variants in iNOS may influence the risk of developing the disorder. We also found an $n N O S$ allele associating with the use of triptans, suggesting an impact on treatment response worth investigating further.

Author Contributions: Conceptualization, A.C.B., data curation, C.F.; formal analysis, C.R.; investigation, C.R. and J.M.M.; methodology, C.F. and J.M.M.; project administration, A.C.B.; resources, C.S., E.W., A.S. and A.C.B.; funding acquisition, A.C.B. and C.R.; supervision, A.C.B.; validation, C.R.; visualization, C.R.; writing—original draft, C.R., and A.C.B.; writing—review and editing, J.M.M., C.F., C.S., E.W., A.S. and A.C.B. All authors read and agreed to the final version of the manuscript.

Funding: This research was funded by the Swedish Brain Foundation and Mellby Gård Foundation (FO2018-0008, FO2019-0004), the Swedish Research Council (2017-01096), Märta Lundqvists stiftelse (2019/2020) and the Karolinska Institutet Foundation and Funds (2018-01738).

Data Availability Statement: The data presented in this study are available on request from the corresponding author. The data are not publicly available due to privacy restrictions.

Acknowledgments: We would like to thank Ann-Christin Karlsson for help with patient recruitment and Armin Tabiei for technical assistance.

Conflicts of Interest: The authors declare no conflict of interest.

\section{References}

1. Nathan, C. Nitric oxide as a secretory product of mammalian cells. FASEB J. 1992, 6, 3051-3064. [CrossRef] [PubMed]

2. The International Headache Society (IHS). Headache Classification Committee of the International Headache Society (IHS) The International Classification of Headache Disorders, 3rd edition. Cephalalgia 2018, 38, 1-211. [CrossRef] [PubMed]

3. Goadsby, P.J.; Edvinsson, L. Human in vivo evidence for trigeminovascular activation in cluster headache Neuropeptide changes and effects of acute attacks therapies. Brain 1994, 117, 427-434. [CrossRef] [PubMed]

4. Hoffmann, J.; May, A. Diagnosis, pathophysiology, and management of cluster headache. Lancet Neurol. 2018, 17, 75-83. [CrossRef]

5. Bredt, D.S. Endogenous nitric oxide synthesis: Biological functions and pathophysiology. Free Radic. Res. 1999, 31, 577-596. [CrossRef]

6. Esplugues, J.V. NO as a signalling molecule in the nervous system. Br. J. Pharmacol. 2002, 135, 1079-1095. [CrossRef]

7. Steinberg, A.; Wiklund, N.P.; Brundin, L.; Nilsson Remahl, A.I.M. Levels of nitric oxide metabolites in cerebrospinal fluid in cluster headache. Cephalalgia 2010, 30, 696-702. [CrossRef]

8. Nemade, R.V.; Lewis, A.I.; Zuccarello, M.; Keller, J.T. Immunohistochemical localization of endothelial nitric oxide synthase in vessels of the dura mater of the Sprague-Dawley rat. Neurosci. Lett. 1995, 197, 78-80. [CrossRef]

9. Berger, R.J.; Zuccarello, M.; Keller, J.T. Nitric oxide synthase immunoreactivity in the rat dura mater. Neuroreport 1994, 5, 519-521. [CrossRef]

10. Thomsen, L.L.; Kruuse, C.; Iversen, H.K.; Olesen, J. A nitric oxide donor (nitroglycerin) triggers genuine migraine attacks. Eur. J. Neurol. 1994, 1, 73-80. [CrossRef]

11. Ashina, M.; Bendtsen, L.; Jensen, R.; Olesen, J. Nitric oxide-induced headache in patients with chronic tension-type headache. Brain 2000, 123, 1830-1837. [CrossRef] [PubMed]

12. Ekbom, K. Nitroglycerin as a Provocative Agent in Cluster Headache. Arch. Neurol. 1968, 19, 487-493. [CrossRef] [PubMed]

13. Sarchielli, P.; Alberti, A.; Codini, M.; Floridi, A.; Gallai, V. Nitric oxide metabolites, prostaglandins and trigeminal vasoactive peptides in internal jugular vein blood during spontaneous migraine attacks. Cephalalgia 2000, 20, 907-918. [CrossRef] [PubMed] 
14. Yilmaz, G.; Sürer, H.; Inan, L.E.; Coşkun, Ö.; Yücel, D. Increased nitrosative and oxidative stress in platelets of migraine patients. Tohoku J. Exp. Med. 2007, 211, 23-30. [CrossRef] [PubMed]

15. Fidan, I.; Yüksel, S.; Ýmir, T.; Irkeç, C.; Aksakal, F.N. The importance of cytokines, chemokines and nitric oxide in pathophysiology of migraine. J. Neuroimmunol. 2006, 171, 184-188. [CrossRef]

16. Shimomura, T.; Murakami, F.; Kotani, K.; Ikawa, S.; Kono, S. Platelet nitric oxide metabolites in migraine. Cephalalgia 1999, 19, 218-222. [CrossRef]

17. Guldiken, B.; Demir, M.; Guldiken, S.; Turgut, N.; Ozkan, H.; Kabayel, L.; Tugrul, A. Asymmetric dimethylarginine and nitric oxide levels in migraine during the interictal period. J. Clin. Neurosci. 2009, 16, 672-674. [CrossRef]

18. Shukla, R.; Barthwal, M.K.; Srivastava, N.; Nag, D.; Seth, P.K.; Srimal, R.C.; Dikshit, M. Blood nitrite levels in patients with migraine during headache-free period. Headache 2001, 41, 475-481. [CrossRef]

19. Costa, A.; Ravaglia, S.; Sances, G.; Antonaci, F.; Pucci, E.; Nappi, G. Nitric Oxide Pathway and Response to Nitroglycerin in Cluster Headache Patients: Plasma Nitrite and Citrulline Levels. Cephalalgia 2003, 23, 407-413. [CrossRef]

20. Alaşehirli, B.; Akçali, A.; Demiryürek, A.T.; Özel, A.; Erdal, M.E.; Neyal, M. Lack of association between the C276T polymorphism of the neuronal nitric oxide synthase gene and migraine. Int. J. Neurosci. 2013, 123, 50-54. [CrossRef]

21. García-Martín, E.; Martínez, C.; Serrador, M.; Alonso-Navarro, H.; Navacerrada, F.; García-Albea, E.; Agúndez, J.A.; JiménezJiménez, F.J. Neuronal nitric oxide synthase (nNOS, NOS1) rs693534 and rs7977109 variants and risk for migraine. Headache 2015, 55, 1209-1217. [CrossRef] [PubMed]

22. Johnson, M.P.; Lea, R.A.; Colson, N.J.; Macmillan, J.C.; Griffiths, L.R. A population genomics overview of the neuronal nitric oxide synthase (nNOS) gene and its relationship to migraine susceptibility. Cell. Mol. Biol. 2005, 51, 285-292. [PubMed]

23. Lea, R.A.; Curtain, R.P.; Shepherd, A.G.; Brimage, P.J.; Griffiths, L.R. No evidence for involvement of the human inducible nitric oxide synthase (iNOS) gene in susceptibility to typical migraine. Am. J. Med. Genet. Neuropsychiatr. Genet. 2001, 105, 110-113. [CrossRef]

24. de OSMansur, T.; Gonçalves, F.M.; Martins-Oliveira, A.; Speciali, J.G.; Dach, F.; Lacchini, R.; Tanus-Santos, J.E. Inducible nitric oxide synthase haplotype associated with migraine and aura. Mol. Cell. Biochem. 2012, 364, 303-308. [CrossRef] [PubMed]

25. Gonçalves, F.M.; Luizon, M.R.; Speciali, J.G.; Martins-Oliveira, A.; Dach, F.; Tanus-Santos, J.E. Interaction among nitric oxide (NO)-related genes in migraine susceptibility. Mol. Cell. Biochem. 2012, 370, 183-189. [CrossRef] [PubMed]

26. Borroni, B.; Rao, R.; Liberini, P.; Venturelli, E.; Cossandi, M.; Archetti, S.; Caimi, L.; Padovani, A. Endothelial nitric oxide synthase (Glu298Asp) polymorphism is an independent risk factor for migraine with aura. Headache 2006, 46, 1575-1579. [CrossRef]

27. Toriello, M.; Oterino, A.; Pascual, J.; Castillo, J.; Colás, R.; Alonso-Arranz, A.; Ruiz-Alegría, C.; Quintela, E.; Montón, F.; Ruiz-Lavilla, N. Lack of association of endothelial nitric oxide synthase polymorphisms and migraine. Headache 2008, 48, 1115-1119. [CrossRef]

28. Dong, H.; Wang, Z.H.; Dong, B.; Hu, Y.N.; Zhao, H.Y. Endothelial nitric oxide synthase (-786T $>$ C) polymorphism and migraine susceptibility. Medicine 2018, 97, e12241. [CrossRef]

29. Gonçalves, F.M.; Martins-Oliveira, A.; Speciali, J.G.; Luizon, M.R.; Izidoro-Toledo, T.C.; Silva, P.S.; Dach, F.; Tanus-Santos, J.E. Endothelial nitric oxide synthase haplotypes associated with aura in patients with migraine. DNA Cell Biol. 2011, 30, 363-369. [CrossRef]

30. Sjöstrand, C.; Modin, H.; Masterman, T.; Ekbom, K.; Waldenlind, E.; Hillert, J. Analysis of nitric oxide synthase genes in cluster headache. Cephalalgia 2002, 22, 758-764. [CrossRef]

31. Steinberg, A.; Fourier, C.; Ran, C.; Waldenlind, E.; Sjöstrand, C.; Belin, A.C. Cluster headache-Clinical pattern and a new severity scale in a Swedish cohort. Cephalalgia 2018, 38, 1286-1295. [CrossRef] [PubMed]

32. Oliveira-Paula, G.H.; Lacchini, R.; Luizon, M.R.; Fontana, V.; Silva, P.S.; Biagi, C.; Tanus-Santos, J.E. Endothelial nitric oxide synthase tagSNPs influence the effects of enalapril in essential hypertension. Nitric Oxide Biol. Chem. 2016, 55, 62-69. [CrossRef] [PubMed]

33. Purcell, S.; Neale, B.; Todd-Brown, K.; Thomas, L.; Ferreira, M.A.; Bender, D.; Maller, J.; Sklar, P.; De Bakker, P.I.; Daly, M.J.; et al. PLINK: A Tool Set for Whole-Genome Association and Population-Based Linkage Analyses. Am. J. Hum. Genet. 2007, 81, 559-575. [CrossRef] [PubMed]

34. Dupont, W.D.; Plummer, W.D., Jr.; Plummer, W.D. Power and sample size calculations. A review and computer program. Control. Clin. Trials 1990, 11, 116-128. [CrossRef]

35. Hougaard, A.; Hauge, A.W.; Guo, S.; Tfelt-Hansen, P. The nitric oxide synthase inhibitor and serotonin-receptor agonist NXN-188 during the aura phase of migraine with aura: A randomized, double-blind, placebo-controlled cross-over study. Scand. J. Pain 2013, 4, 48-52. [CrossRef]

36. Bhatt, D.K.; Gupta, S.; Jansen-Olesen, I.; Andrews, J.S.; Olesen, J. NXN-188, a selective nNOS inhibitor and a 5-HT1B/1D receptor agonist, inhibits CGRP release in preclinical migraine models. Cephalalgia 2013, 33, 87-100. [CrossRef]

37. De Felice, M.; Ossipov, M.H.; Wang, R.; Dussor, G.; Lai, J.; Meng, I.D.; Chichorro, J.; Andrews, J.S.; Rakhit, S.; Maddaford, S.; et al. Triptan-induced enhancement of neuronal nitric oxide synthase in trigeminal ganglion dural afferents underlies increased responsiveness to potential migraine triggers. Brain 2010, 133, 2475-2488. [CrossRef] 